\title{
Defining critical parameters in a luminescent downshifting layer for PV enhancement
}

\author{
Maria F. Ayala Barragan ${ }^{1}$, Subhash Chandra ${ }^{1}$, Bill Cass ${ }^{1}$, Sarah J. McCormack ${ }^{1}$ \\ ${ }^{1}$ Department of Civil, Structural and Environmental Engineering, School of Engineering, Trinity College \\ Dublin, College Green, Dublin 2, IRELAND \\ Corresponding author email: mccorms1@tcd.ie
}

\begin{abstract}
This paper presents the study and evaluation of luminescent down shifting (LDS) layers doped with organic dyes and their compatibility with different types of solar cells. In this study commercial dyes in the Lumogen-F series (BASF) Violet 570, Orange 240, Red 300, Yellow 083, and Yellow 170 were considered for this analysis. It was found that while organic dyes fulfil important characteristics for luminescent materials for LDS such as high photoluminescent quantum yield, small radiative overlap, absorption in or close to the UV range and emission in the visible spectrum. Nevertheless, due to their low Stokes Shift, organic dyes do not entirely align to the spectral response of solar cells as the spectral matching of the emission and absorption are characteristics that depend on the external quantum efficiency of the solar cell. In this paper a design matrix was developed for the commercial dyes outlined and different solar cell types. For different parameters of the luminescent materials and solar cells the optimized LDS layer can be chosen.
\end{abstract}

KEYWORDS: Downshifting, Luminescence, Downshifting Layers, Photovoltaics

\section{INTRODUCTION}

While there are several loss mechanisms within a solar cell, an important aspect to consider is the low efficiency of solar cells when absorbing high energy photons. Luminescent downshifting layers (LDS) is a novel way of improving the efficiency of solar cells under high energy ultraviolet radiation $[1,2,3]$. LDS consists of a layer that absorbs high energy photons and converts them to a lower energy where the solar cell is able to absorb and convert them to electricity more efficiently. The LDS process is enabled by installing a thin polymer layer on top of the solar cell thus avoiding the need for performing any internal structural changes on solar cells which could hinder their performance or damage them. Figure 1 is a representation of the optical processes involved in the LDS layer as well as the losses that occur due to the layer on top of the solar cell.

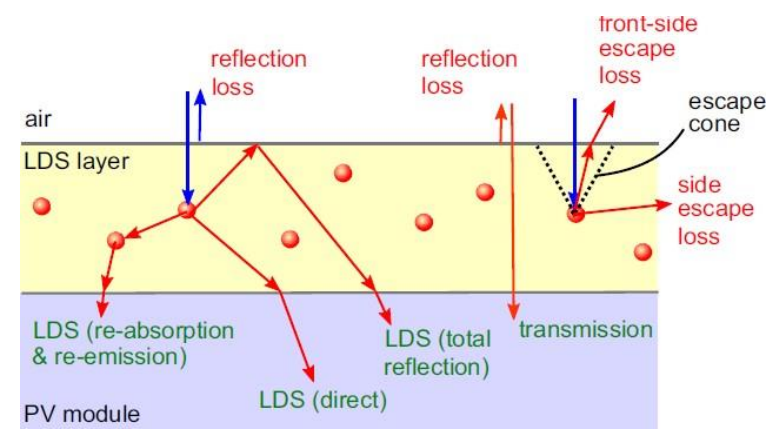

Figure 1 LDS optical process and losses [4]

\section{METHODOLOGY}

The luminescent and host materials used in LDS layers have several characteristics that must be carefully selected in order to achieve the maximum potential of the layer. Commercial dyes in the Lumogen $-F$ series. Dyes Violet 570, Orange 240, Red 300, Yellow 083, and Yellow 170 were considered for this analysis. Lumogen-F dyes are produced by (BASF). Dyes O240, R300, Y083 and Y170 are Perylene based while V570 is Napthalimide based. While it has been shown that Perylene based dyes have great stability, both types of dyes have high heat stability which is necessary in dyes used in any type of solar cell or solar concentrator [5]. Lumogen-F dyes are easily incorporated into polymer sheets 
and have strong luminosity. These dyes are easily soluble in toluene, acetone, isopropyl alcohol, ethyl acetate, xylene among others [6].

Using data from [7], the normalised absorption and emission spectra of the Lumogen-F series organic dyes was obtained. The data was then used to calculate the Stokes Shift and the overlap between the absorption and emission spectra in order to analyse which dye performs best according to the se characteristics. Figure 2 presents the normalised absorption and emission spectra for all Lumogen dyes (R300, Y170, V570, O240 \& Y083) along with the overlap and the Stokes Shift (SS) (the difference between positions of the maxima of the absorption and emission spectra).

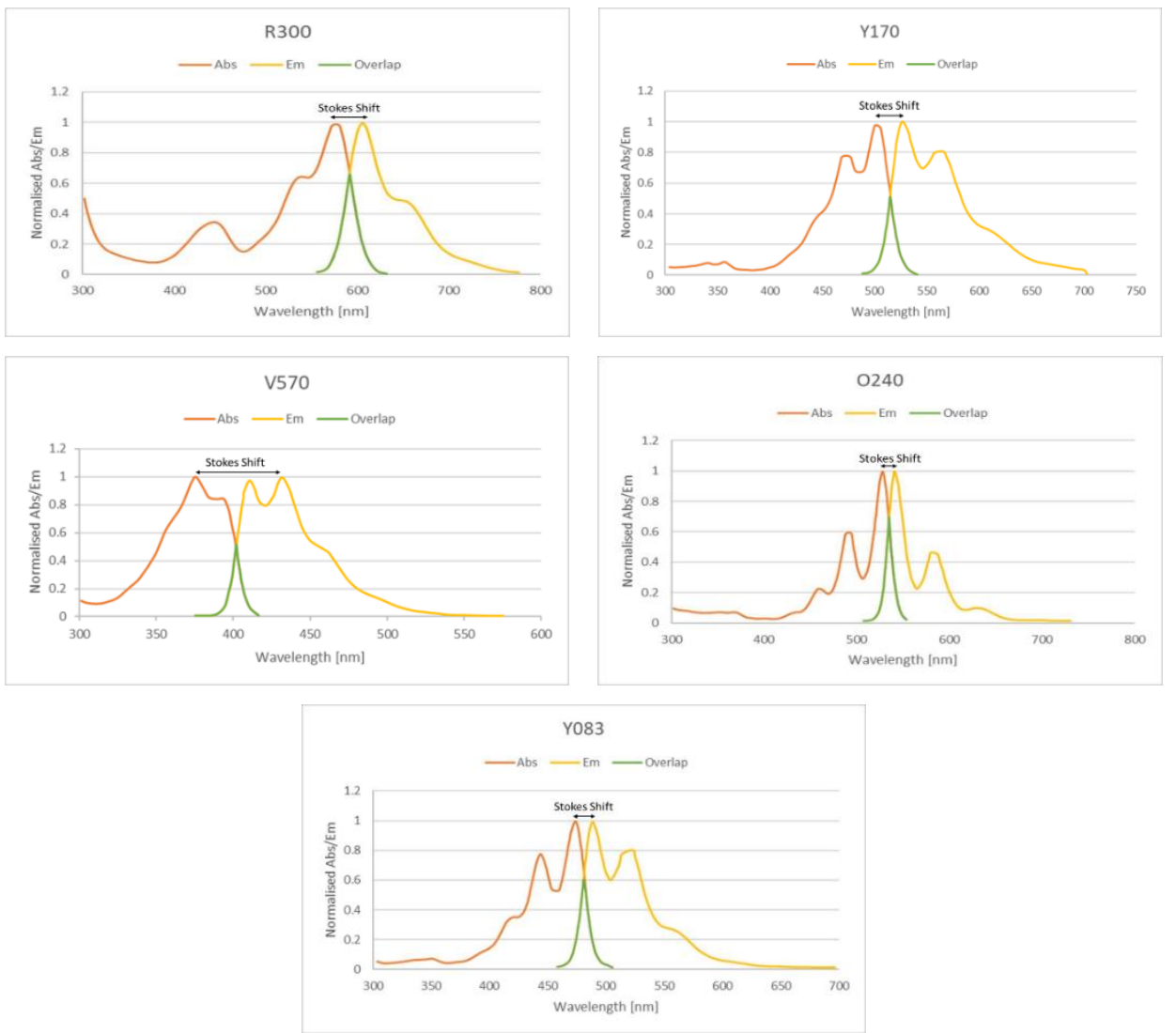

Figure 2 Absorption, Emission and Overlap of dye R300, Y170, V570, O240 \& Y083

Figure 3 depicts the values of the radiative overlap, SS and PLQY of every dye. R300 has the highest radiative overlap (RO) and one of the highest SS. V570 shows excellent characteristics with the smallest RO and the largest SS of all the dyes. Organic dyes do not have a large SS leading to overlap between the absorption and emission bands. This characteristic has allowed for rare earth phosphors (REPs) to be used by taking advantage of their sharp spectra bands. When comparing QDs to organic dyes, QDs absorb in narrow bands generally localised where the SR of the solar cell is low and emit where the EQE of cells is high. Nevertheless, organic dyes have higher PLQY than QDs ultimately being able to convert the energy absorbed more efficiently than QDs. Low PLQY in commercial QDs renders them a less attractive luminescent material for LDS layers. 


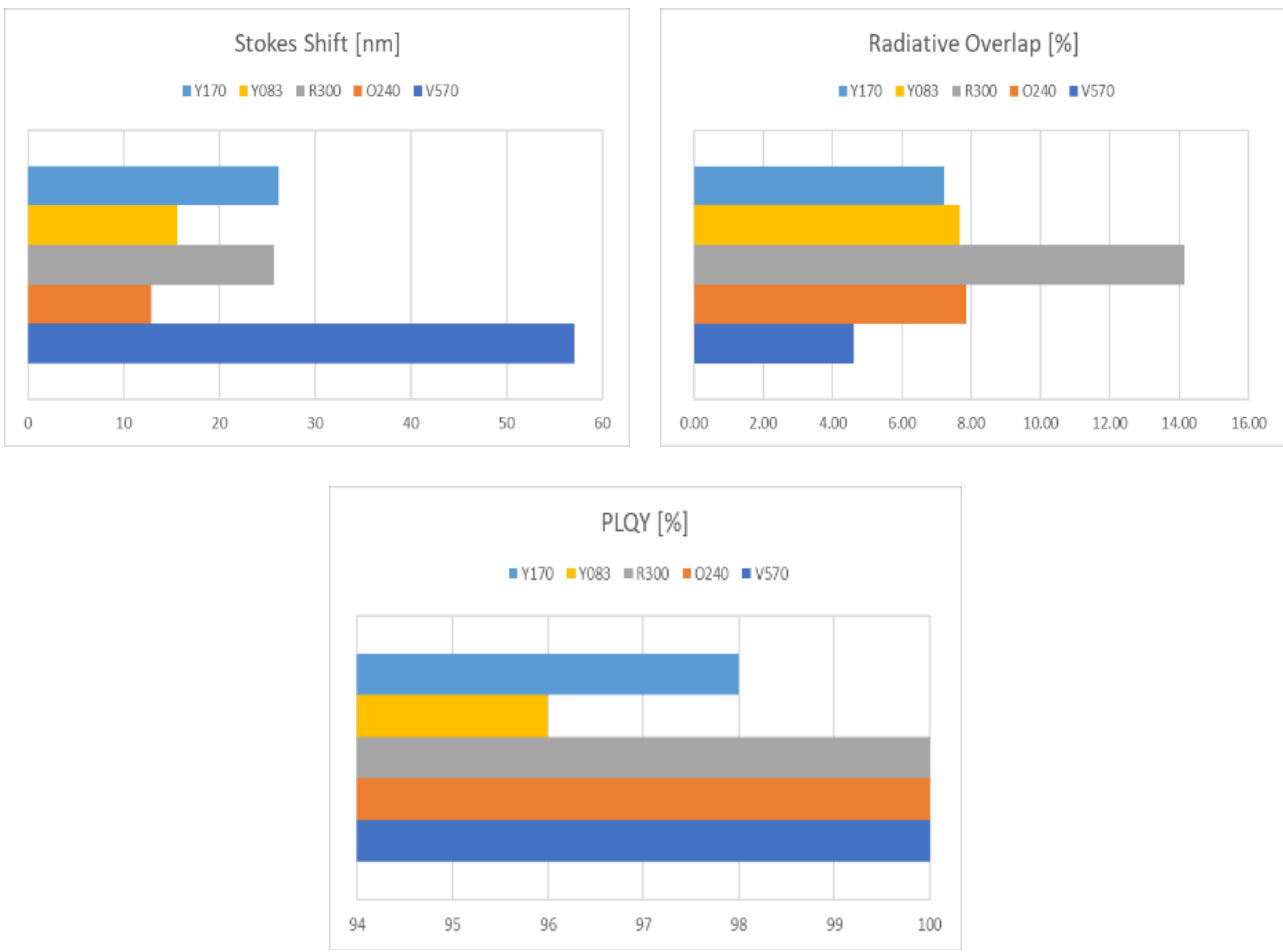

Figure 3 PLQY, Radiative Overlap and Stokes Shift of Lumogen Dyes

In table 1, the characteristics of organic dyes as a luminescent material are explored and organised in a colourcoded matrix based on their importance and effect on the performance: Green = Excellent characteristic, Yellow = Satisfactory characteristic; Red $=$ Undesirable characteristic.

Table 1 Lumogen Dye Performance Matrix

\begin{tabular}{|c|c|c|c|c|c|}
\hline & $\begin{array}{c}\text { Max Abs } \\
(\mathrm{nm})\end{array}$ & $\begin{array}{c}\text { Max Em } \\
(\mathrm{nm})\end{array}$ & $\begin{array}{c}\text { Stokes Shift } \\
(\mathrm{nm})\end{array}$ & $\begin{array}{c}\text { Radiative Overlap } \\
(\%)\end{array}$ & PLQY (\%) \\
\hline V570 & 375.92 & 432.98 & 57.06 & 4.62 & 100 \\
\hline 0240 & 527.77 & 540.66 & 12.89 & 7.86 & 100 \\
\hline R300 & 579.81 & 605.47 & 25.66 & 14.16 & 100 \\
\hline Y083 & 473.19 & 488.76 & 15.57 & 7.67 & 96 \\
\hline Y170 & 500.82 & 526.99 & 26.17 & 7.20 & 98 \\
\hline
\end{tabular}

The above decision matrix was created by analysing solely the characteristics of the Lumogen- $F$ series of organic dyes as luminescent materials. Each characteristic was ranked independently from each other and based on the literature it was established whether it was an acceptable characteristic or not. It is clear, based on Table 3.2, that dyes V570 and Y083 are the dyes which show the best characteristics. Unfortunately, dye V570 has been removed from the market and is not available for purchase anymore. As previously discussed, dye Y083 had an excellent performance due to its properties showing satisfactory absorption and emission spectra, high PLQY, and low RO. Nevertheless, even when luminescent materials show excellent properties previously discussed, the SR of a solar cell plays an important role in the performance of the LDS layer. If the emission of the dye falls within the poor SR range of the cell, the layer will not have a notorious improvement in its EQE and could even provoke further losses due to reabsorption.

In Figure 4 the SR of different solar cells was plotted against the absorption and emission of each of the Lumogen-F dyes. The solar cells analysed and plotted are c-Si, mc-Si, CdTe, CIGS, DSSC, OSC, and Gallium Arsenide (GaAs). Some dyes may have great ASM and/or ESM for one type of cell but poorly match another. Such is the case of dye V570 with Si solar cells and CdTe cells respectively. While dye V570 has good ASM with Si cells, it is better with CdTe cells than with either mono or poly-crystalline Si. However, V570 has a poor ESM as it emits at a range that falls where the SR of CdTe cells is still poor. Similarly, there are cells that are not compatible with the Lumogen- $\mathrm{F}$ dye series as all dyes absorb in a range where the cell has a high spectral 
response thus, adding a layer would decrease the EQE of the cell. From the graphs in Figure 4, it can be seen that the SR of DSSC and OSC illustrate how dyes may not be the best luminescent material for this type of cells as they absorb where the SR of the cell is close to its highest peak.
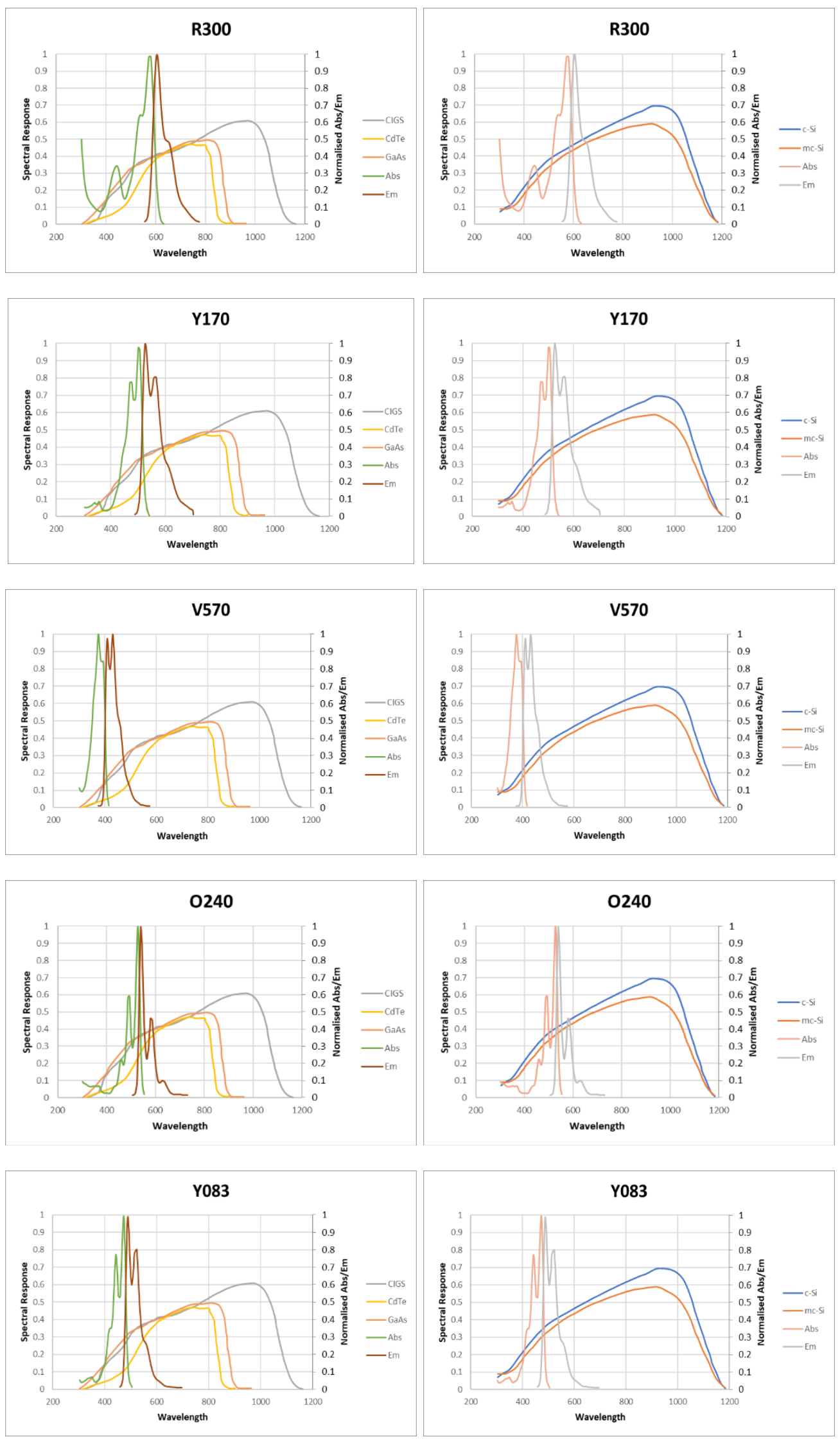

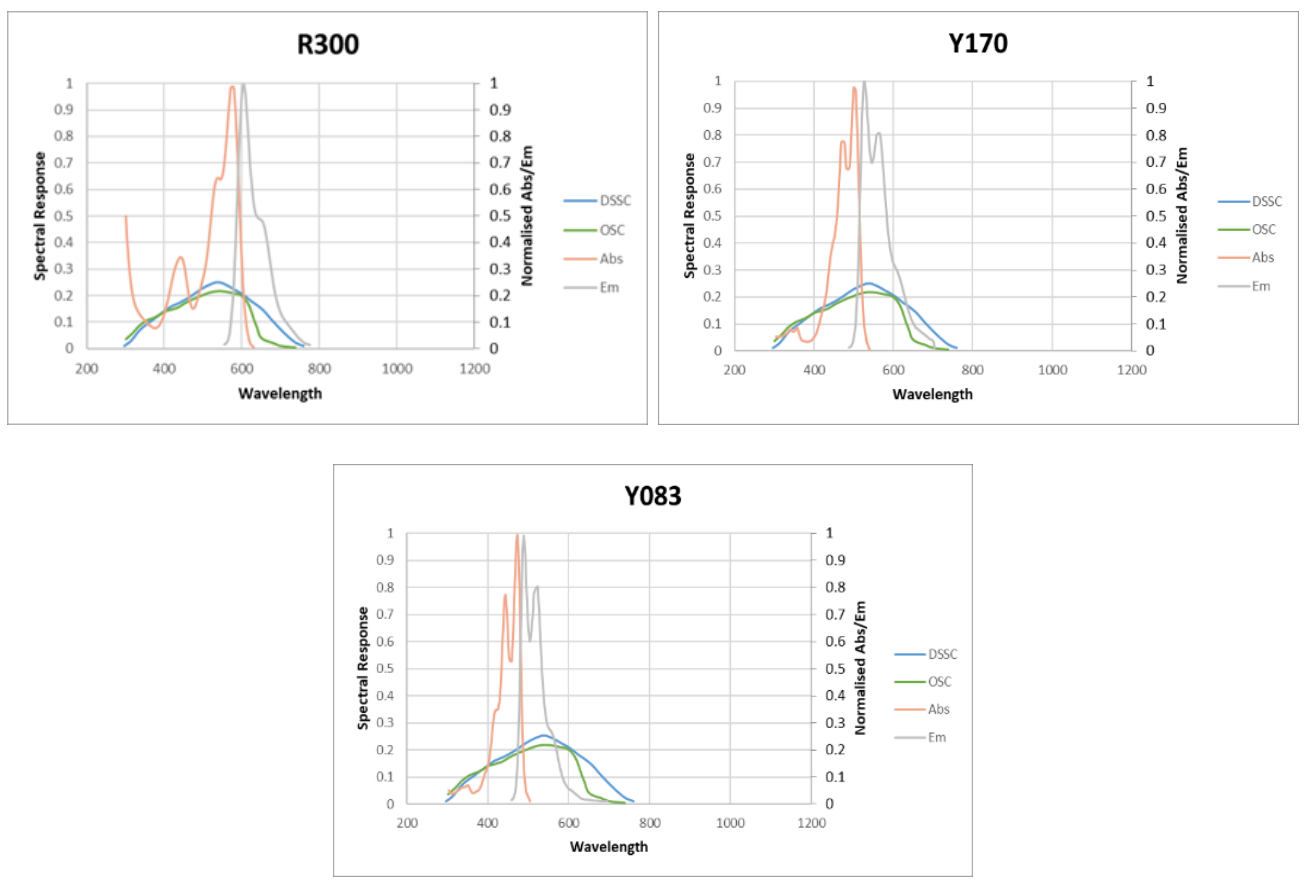

Figure 4 Absorption and Emission profiles with Spectral Response

The maximum values of absorption and emission may give a sense of how well a dye matches a cell, but it is not enough to analyse how compatible the dye is with a type of cell. For this, the complete absorption and emission profiles of each dye were plotted against five types of cells (c-Si, mc-Si, CdTe, CIGS, DSSC, OSC, and $\mathrm{GaAs}$ ) in order to calculate the overlap each spectrum has with the spectral response. The percentage overlap between absorption or emission and the SR of a solar cell was calculated to better visualise the potential behaviour of the dye with the cell.

When analysing absorption and emission spectra, it is expected that the absorption does not interfere with the solar cell absorbing photons i.e., the dye absorbs in a range where the cell has poor to no SR. At the same time, the same dye has to emit the downshifted photons back to the cell at a range where the SR is at its maximum. The overlap between absorption and SR should be low in order to avoid parasitic absorption. Contrarily, the overlap between emission and SR should be high, meaning that the downshifted photons have a higher chance to be absorbed and be efficiently used by the solar cell.

Dye V570 was an excellent option to use with Si solar cells due to its absorption in the UV region as seen in Figure 3.10 above. However, the emission is still far away from an optimal SR of other solar cells making it less advantageous for solar cells such as CdTe. Dye R300 does not have an optimal absorption overlap as its highest peak starts far in the visible range, where most solar cells already have a good absorption. In theory, it could work well for solar cells with high SR in the late visible spectrum such as CIGS, but its absorption interferes with the SR potentially causing PA. The graphs in Figure 3.10, depict how dye R300 absorbs at ranges where the solar cell SR is already high and emits either at a good wavelength where the SR is high as seen for CIGS or emits where the SR is already declining as seen for OSC. Similarly, dyes Y083 and Y170 have been mentioned in the literature as dyes with good EQE responses when used in LDS layers. Dye O240 has a small SS and an absorption far in the visible range which could cause PA in the solar cell if used as luminescent material. It is especially not the best choice for OSC and DSSC as its absorption spectrum hinders the SR of these cells as seen in in the graphs above.

The tables below show the percentage overlap between the dye spectrum and the SR of the cell. Table 3.3 only considers absorption values while Table 3.4 only considers emission values. Table 3.5 shows a merged version of Tables 3.3 and 3.4 with its own colour-code. It does not contain values since they are represented in the tables above. This table is a visual summary of Tables 3.3 and 3.4. No dye has an optimal overlap for 
both absorption and emission. Thus, if a dye had an optimal overlap for either absorption or emission, it was considered as optimal (green) in the final table. If both absorption and emission are sub-optimal, then in Table 3.5, it was considered as sub-optimal (yellow). Finally, if one of either absorption or emission was non-optimal, regardless of the classification of the other value, then in Table 3.5 it was considered non-optimal (red) as well.

For absorption: Green = 0-25\% (optimal percentage overlap); Yellow $=25-50 \%$ (sub-optimal percentage overlap); Red = 50\% (non-optimal percentage overlap). For emission: Green = 50\% (optimal percentage overlap); Yellow = 50-25\% (sub-optimal percentage overlap); Red = 25-0\% (non-optimal percentage overlap).

Tables 3.3-3.4 Lumogen Dye Absorption and Emission Overlap

\begin{tabular}{|c|c|c|c|c|c|}
\hline $\begin{array}{c}\text { Absorption } \\
\text { Overlap (\%) }\end{array}$ & V570 & O240 & R300 & Y083 & Y170 \\
\hline c-Si & 15.13 & 37.94 & 75.63 & 32.18 & 40.95 \\
\hline CIGS & 5.2 & 32.03 & 61.68 & 22.34 & 30.56 \\
\hline GaAs & 8.25 & 32.71 & 65.01 & 26.51 & 34.73 \\
\hline OSC & 10.92 & 25.85 & 48.06 & 21.7 & 27.07 \\
\hline DSSC & 9.87 & 27.71 & 50.63 & 22.43 & 28.38 \\
\hline CdTe & 2.72 & 19.96 & 42.11 & 10.02 & 14.77 \\
\hline mc-Si & 12.68 & 37.11 & 70.89 & 28.75 & 37.35 \\
\hline
\end{tabular}

\begin{tabular}{|c|c|c|c|c|c|}
\hline $\begin{array}{c}\text { Emission } \\
\text { Overlap (\%) }\end{array}$ & V570 & O240 & R300 & Y083 & Y170 \\
\hline c-Si & 28.92 & 33.64 & 54.90 & 39.34 & 52.65 \\
\hline CIGS & 22.22 & 31.83 & 50.02 & 35.54 & 48.83 \\
\hline GaAs & 24.47 & 31.49 & 50.09 & 36.14 & 48.32 \\
\hline OSC & 19.53 & 22.47 & 13.86 & 26.35 & 28.33 \\
\hline DSSC & 20.51 & 24.50 & 23.06 & 28.72 & 34.51 \\
\hline CdTe & 11.43 & 28.56 & 48.92 & 25.80 & 41.80 \\
\hline mc-Si & 25.71 & 32.44 & 52.93 & 36.92 & 50.08 \\
\hline
\end{tabular}

Table 3.5 Lumogen Dye Performance Decision Matrix

\begin{tabular}{|c|c|c|c|c|c|}
\hline & V570 & 0240 & R300 & Y083 & Y170 \\
\hline C.Si & & & & & \\
\hline CIGS & & & & & \\
\hline GaAs & & & & & \\
\hline OSC & & & & & \\
\hline DSSC & & & & & \\
\hline CdTe & & & & & \\
\hline mc.Si & & & & & \\
\hline
\end{tabular}

These matrices provide an estimated representation of the performance dyes have on different solar cells and are not an absolute evaluation of their behaviour. They confirm the analysis made on dye V 570 being a good match for c-Si but incompatible withCdTe. With these matrices, it is clearer to compare the SR to the spectrum of each dye. The analysis was made based solely on the absorption and emission spectra of the dyes compared with the SR of different solar cells.

\section{CONCLUSION}

After thoroughly analysing the characteristics of organic dyes as luminescent materials, it is concluded that organic dyes fulfil important characteristics for luminescent materials for LDS such as high PLQY, small RO, absorption in or close to the UV range and emission in the visible spectrum. Nevertheless, due to their low SS, organic dyes do not entirely align to the SR of solar cells as ESM and ASM are characteristics that depend on the EQE of the solar cell. The concentration of the dye in the layer also plays an important role in the EQE of the cell with an LDS layer. A highly concentrated layer may cause PA and lower the EQE of the cell while a layer with a small concentration of dye may not be able to absorb and downshift enough photons to change the EQE of the cell. No dye will match all necessary characteristics nor will it be a perfect match for all types of solar cells. It is the combination of all their characteristics that make them great luminescent materials and an ideal choice for LDS layers.

\section{REFERENCES}

1. Klampaftis, E., Ross, D., Mclntosh, K. R. \& Richards, B. S., (2009). Enhancing the performance of solar cells via luminescent down-shifting of the incident spectrum: A review. Solar Energy Materials and Solar Cells 93, 1182-1194.

2. Ahmed, H., Doran, J., \& S.J. McCormack., (2016). Increased short-circuit current density and EQE of silicon and dye sensitised solar cells through plasmonic LDS layers, Solar Energy, V. 126, pp 146-155, https://doi.org/10.1016/j.solener.2016.01.003.

3. Ahmed, H., Doran, J., \& S.J. McCormack., (2017). Plasmonic luminescent down shifting layers for the enhancement of CdTe mini-modules performance, Solar Energy, V. 141, pp 242-248, 
4. Rothemund, R., (2014). Optical modelling of the external quantum efficiency of solar cells with luminescent down-shifting layers. Solar Energy Materials \& Solar Cells, 120, 6.

5. Ross, D., Alonso-Alverez, D., Klampaftis, E., Fritsche, J., Bauer, M., Debije, M. G., Fifield, R. M. \& Richards, B. S., (2014). The Impact of Luminescent Down Shifting on the Performance of CdTe Photovoltaics: Impact of the Module Vintage. IEEE Journal of Photovoltaics 4, 8.

6. BASF, (1997). Lumogen F Technical Information Colorants and Graphic Systems.

4. Alonso-Alvarez, D., Ross, D. \& Richards, B. S., (2012). Luminescent down-shifting for CdTe solar cells: A review of dyes and simulation of performance. 8th IEEE Photovoltaic Specialists Conference Photovoltaic Specialists Conference (PVSC). Austin, TX, USA.

7. Crevant, C., Lucchesi, C., Paire, M. \& Guilmmemoles, J.-F. O., (2017). LDS Layers Using Organic Dyes and Quantum Dots. Physica Status Solidi C, 14, 13-20. 